\section{Accuracy amidst ambiguity: false positive SARS-CoV-2 nucleic acid tests when COVID-19 prevalence is low}

Sir,

In countries with a low prevalence of COVID-19 and a low pre-test probability, confirmation of positive nucleic acid test (NAT) results for SARS-CoV-2 is recommended given the potential for false positive results.

As of 24 September 2020, there have been 26,983 confirmed cases and 861 deaths from COVID-19 in Australia. Widespread testing, together with Australia's geographic advantage, border controls, social distancing and public health messaging have all contributed to limit the number of infections. Australia has one of the highest testing rates in the world with $7,441,327$ SARS-CoV-2 NATs performed on $6.4 \%$ of the population ${ }^{1}$ since 22 January 2020 , of which $0.4 \%$ were positive. The prevalence of laboratoryconfirmed COVID-19 has varied between the different jurisdictions in Australia since the pandemic was declared, with the highest overall rate of $0.8 \%$ in Victoria in July 2020.

During the early phases of the pandemic, SARS-CoV-2 NATs were mainly performed by public health laboratories using 'in-house' developed tests targeting one or more regions of the SARS-CoV-2 genome. Over time, commercial NATs became available, and testing was also undertaken by private laboratories. Ideally and prior to intended use, all SARS-CoV-2 diagnostic assays should be validated to ensure they are fit for purpose. The urgent nature of the pandemic led to expedited assessments of many SARS-CoV-2 tests by regulatory bodies such as the Therapeutic Goods Association in Australia and the Food and Drug Administration in the United States of America, with approvals contingent on the supply of ongoing evidence to support the safety and performance of the assays. ${ }^{2-4}$ Initially, the Public Health Laboratory Network (PHLN) Australia recommended that confirmatory testing be performed on samples where SARSCoV-2 RNA had been detected to ensure that the result was a true positive. ${ }^{5}$ However, this was not always practical or efficient with substantial testing volumes and in the face of shortages of nucleic acid extraction and testing reagents and consumables.

Despite the second wave of infections in Victoria, the prevalence of COVID-19 in Australia remains low $(<1 \%)$, meaning that in the absence of epidemiological risk factors, the pre-test probability will be low and false positive results will occur even with highly specific NATs. For example, the positive predictive value (PPV) of SARS-CoV-2 NATs with a specificity of $99 \%$ is only $50 \%$ when the prevalence of infection is $1 \% .^{6}$ At the height of the pandemic in Australia in late March, the prevalence of COVID-19 was 2\% (range $0.7-3.4 \%$ between the different States and Territories), indicating that NATs with the same analytical performance will have a PPV of approximately $67 \%$ (range $41.2-77.3 \%$ ).

When SARS-CoV-2 was detected by in-house or commercial NATs in NSW Health Pathology (NSWHP) laboratories, samples were sent to NSWHP-Institute of Clinical
Pathology and Medical Research, Westmead Hospital, for supplementary testing as part of NSWHP's testing algorithm to minimise false positive results. Supplementary testing was performed using real time reverse-transcriptase polymerase chain reaction (RT-PCR) assays targeting the E, RdRp, M, N, ORF1ab and ORF1b genes ${ }^{8}$ using either referred nucleic acid extract and/or nucleic acid re-extracted from the original sample using the MagNA Pure 96 instrument (Roche Diagnostics, Germany). The final result was determined as the consensus of the results from testing the six targets above. Of 122 samples referred from both internal and external laboratories for SARS-CoV-2 confirmatory testing from 14 July to 24 September 2020, we identified a false positive rate of $11 \%$ (13/122). Only two of the 13 cases defined as false positive had SARS-CoV-2 serology and/or respiratory tract PCR results available, both testing negative for SARS-CoV2-specific serology and one patient testing positive for rhinovirus.

False positive results may not always be easily identified, and laboratory staff (alone, or in conjunction with clinicians and/or public health physicians) should remain vigilant for their presence. Suspicion should arise if there are discrepant clinico-epidemiological findings (particularly problematic when there are many asymptomatic infections); unexpected laboratory results (such as discordant results where only one SARS-CoV-2 target is detected in assays with multiple targets, and/or RT-PCR results with high cycle threshold values) or contamination (for example, when a batch of samples test positive); incorrect results from external quality assurance programs; warnings from diagnostic companies about potential contaminated assays or reagents ${ }^{9}$; or when supplemental NATs on other platforms, the use of different SARS-CoV-2 targets, SARS-CoV-2-specific serology, or genomic sequencing do not concur with the initial NAT result.

In the context of Australia's low prevalence of COVID-19 and thus low pre-test probability for infection, we recommend that all positive SARS-CoV-2 NAT results be confirmed by supplementary testing on the original nucleic acid extract and/or re-extraction of nucleic acid from the original sample (if available) and tested using another assay(s) with different gene targets and/or lower limits of detection $^{10}$ (Fig. 1). Repeat respiratory tract sampling (including sputum if available), especially in asymptomatic individuals with no identified epidemiological links, is an approach that has been implemented by laboratories following the release of the PHLN guidance document on NAT result interpretation for SARS-CoV-2. ${ }^{6}$ Sera should also be collected for the detection of SARS-CoV-2-specific antibodies (Fig. 1). Serology testing may not always confirm acute SARS-CoV-2 infection (particularly if collected early in the illness course, as SARS-CoV-2-specific antibodies appear around 10 days after disease onset) but can be useful if positive. However, confirmation of infection requires convalescent sera to be collected to demonstrate seroconversion or a four-fold or greater rise in antibody titres between the acute and convalescent samples. Serology may also be used for retrospective diagnosis of SARS-CoV-2 where NAT testing was not performed or was inconclusive. A combination of these approaches will assist with the 


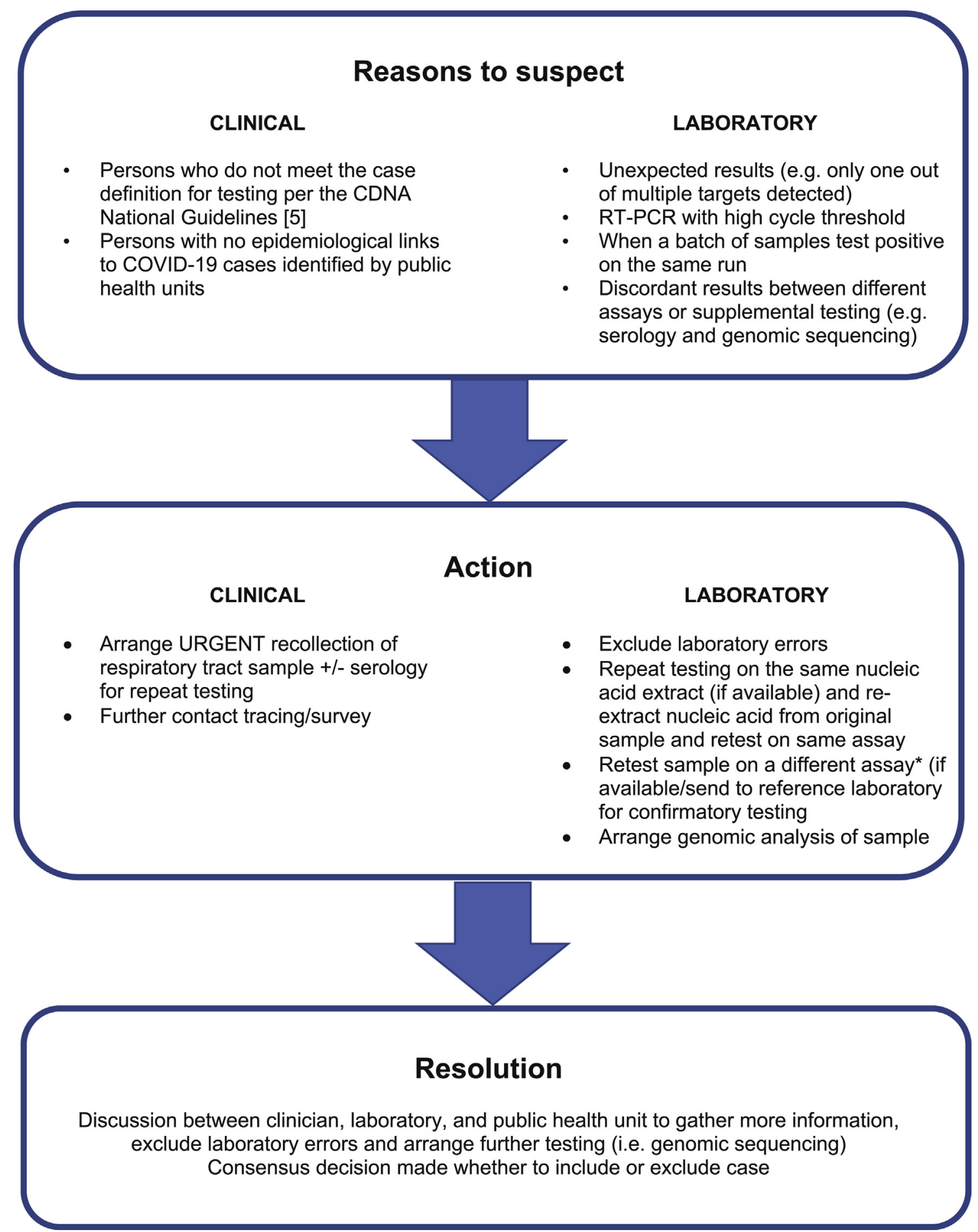

Fig. 1 Approach to investigating suspected false positive SARS-CoV-2 results. *The testing should be repeated on an assay that is of equal or superior sensitivity.

recognition of reinfection, with SARS-CoV-2-specific antibody assays still to be validated. ${ }^{11}$

Timely identification of true false positive SARS-CoV-2 NAT results is important as unrecognised false positive results can lead to unnecessary quarantining and contact tracing, delays in the recognition and treatment of the true illness, significant patient anxiety and concern, potential exposure to nosocomial infection from other patients with confirmed COVID-19, wastage of personal protective equipment, and inaccurate statistics regarding local prevalence of infection.
Conflicts of interest and sources of funding: The authors state that there are no conflicts of interest to disclose.

\section{K. Basile ${ }^{1}$, S. Maddocks ${ }^{1}$, J. Kok ${ }^{1}$, D. E. Dwyer ${ }^{1,2}$}

${ }^{1}$ Centre for Infectious Diseases and Microbiology Laboratory Services, NSW Health Pathology-Institute of Clinical Pathology and Medical Research, Westmead Hospital, Westmead, NSW, Australia; ${ }^{2}$ Marie Bashir Institute for Infectious Diseases and Biosecurity, The University of Sydney, Sydney, NSW, Australia 
Contact Dr Kerri Basile.

E-mail: kerri.basile@ health.nsw.gov.au

1. Australian Government Department of Health. Coronavirus (COVID19) current situation and case numbers. Cited 20 Sep 2020. https://www. health.gov.au/news/health-alerts/novel-coronavirus-2019-ncov-healthalert/coronavirus-covid-19-current-situation-and-case-numbers\#testsconducted-and-results.

2. Therapeutic Goods Association. COVID-19 testing in Australia - information for health professionals. COVID-19 test performance. Cited 17 Jun 2020. https://www.tga.gov.au/covid-19-testing-australia-information-health-professionals

3. Woloshin S, Patel N, Kesselheim AS. False negative tests for SARS-CoV 2 infection - challenges and implications. N Engl J Med 2020; 383: e38.

4. US Food and Drug Administration. Emergency Use Authorization (EUA) information, and list of all current EUAs. Cited 17 Jun 2020. https://www.fda.gov/emergency-preparedness-and-response/mcm-legalregulatory-and-policy-framework/emergency-use-authorization

5. Australian Government Department of Health. PHLN guidance on laboratory testing for SARS-CoV-2 (the virus that causes COVID-19). Cited 8 Jun 2020. https://www.health.gov.au/resources/publications/ phln-guidance-on-laboratory-testing-for-sars-cov-2-the-virus-thatcauses-covid-19

6. Australian Government Department of Health. PHLN guidance on nucleic acid test result interpretation for SARS-CoV-2. Cited 19 Sep 2020. https://www health gov au/resources/publications/phln-guidanceon-nucleic-acid-test-result-interpretation-for-sars-cov-2

7. COVID-19 National Incident Room Surveillance Team. COVID-19, Australia: Epidemiology Report 9 (Reporting week to 23:59 AEDT 29 March 2020). Commun Dis Intell 2020; 44: https://doi.org/10.33321/ cdi.2020.44.29.

8. Rahman H, Carter I, Basile K, et al. Interpret with caution: an evaluation of the commercial AusDiagnostics versus in-house developed assays for the detection of SARS-CoV-2 virus. J Clin Virol 2020; 127: 104374.

9. Bustin SA, Nolan T. RT-qPCR testing of SARS-CoV-2: a primer. Int $J$ Mol Sci 2020; 21: E3004.

10. US Food and Drug Administration. SARS-CoV-2 reference panel comparative data. Cited 21 Sep 2020. https://www.fda.gov/medicaldevices/coronavirus-covid-19-and-medical-devices/sars-cov-2reference-panel-comparative-data

11. European Centre for Disease Prevention and Control. Threat Assessment Brief. Reinfection with SARS-CoV: considerations for public health response. 21 Sep 2020. https://www.ecdc.europa.eu/sites/default/files/ documents/Re-infection-and-viral-shedding-threat-assessment-brief.pdf

DOI: https://doi.org/10.1016/j.pathol.2020.09.009

\section{The impact of viral transport media on PCR assay results for the detection of nucleic acid from SARS-CoV-2}

Sir,

For several decades a variety of medium solutions have been recommended to stabilise specimens for the detection of bacteria and viruses, particularly during diagnostic investigations. These have usually been based on balanced salt or saline solutions with a buffering capacity to maintain a 'near-neutral' $\mathrm{pH}$. To enhance the stability of viruses a spectrum of protein supplements has and continues to be recommended. ${ }^{1-3}$ While some laboratories have prepared viral transport medium (VTM) 'in house', commercial preparations are used extensively and are often supplied as part of a sample collection kit with sterile swabs. Testing of samples by cultural methods meant that the emphasis of studies for the evaluation of these products originally focussed on the capacity of a preparation to maintain the infectivity of viruses at different temperatures while being held prior to and during transport and while being stored at the laboratory. With the widespread introduction of molecular based diagnostic assays, especially real time PCR (qPCR), studies have been undertaken to evaluate the stability of viruses in VTMs, particularly in commercially prepared products, while being held at a range of temperatures. ${ }^{4,5}$ However, while thermal stability has been considered, generally little attention has been given to other characteristics of the VTM or the potential impact of endogenous components. One commercially available product is specifically designed to inactivate viruses and bacteria and contains components to inhibit the activity of nucleases that may be present in the sample. ${ }^{6}$ There are some other products that are recommended for use in molecular detection assays but the manufacturers provide no comment that these products are unlikely to be suitable for samples where virus culture will be attempted.

During large-scale disease epidemics there can be pressure placed on the capacity of manufacturers to supply transport media and, during a pandemic, supply-chain and manufacturing pressures can become prohibitive. During the current (2020) SARS-CoV-2 pandemic, there has been an acute shortage of VTM in Australia because of a combination of both local and international demand, the lack of a local manufacturer and partly because of reduced international airline flights to Australia. Consequently, many different VTMs and similar solutions have been used to meet the demand for transport media generated by large scale diagnostic and surveillance testing. After becoming aware of concerns of variable results for the same samples in different assays, we initiated a study to compare the stability of SARSCoV-2 RNA in several commercially manufactured VTMs and an in-house product. The commercial products were UTM-RT (Copan, Italy), Citoswab (Citotest Labware, China) and CP VTF (Edwards, Australia), while the in-house product (VTM-1) was based on phosphate buffered saline (PBS $\mathrm{pH}$ 7.2) supplemented with $0.5 \%$ gelatin (PBGS). The commercial products are believed to be supplemented with bovine serum albumen and gelatin or bovine serum. Sterile phosphate buffered saline (PBS), $\mathrm{pH} 7.2$, was used as a control. Within 30-45 minutes of preparation, the dilutions of RNA in each VTM were extracted and tested by semiquantitative real time PCR (qRT-PCR). Subsequently, this pilot experiment was repeated under the same conditions with a series of dilutions of a high titred patient sample (P66) which had been recently collected and with a Type A influenza virus and the RNA extracted from it.

Total nucleic acid was extracted from $50 \mu \mathrm{L}$ of each sample with a magnetic bead-based viral RNA extraction kit (MagMax96 Viral RNA; Ambion, USA) run on a Kingfisher96 magnetic particle handling system (ThermoFisher, USA). The nucleic acid was eluted in $50 \mu \mathrm{L}$ and $5 \mu \mathrm{L}$ run in an in house qRT-PCR. Primers and probes were directed at the SARS-CoV-2 E gene ${ }^{7}$ and the RdRp gene. ${ }^{8}$ These SARS$\mathrm{CoV}-2$ primers and probes were used in a triplex assay with the inclusion of an exogenous RNA internal control (XIPC) assay. ${ }^{9}$ This XIPC RNA (approximately 80 copies/ $\mu \mathrm{L})$ was included in the sample lysis buffer prior to the extraction of nucleic acid. A commercial reverse transcriptase mastermix (AgPath-ID One-Step RT-PCR kit; Life Technologies, USA) was used for the qRT-PCR and run on an ABI7500 or Quantstudio 5 (ThermoFisher Scientific, USA) thermocycler. The design of this study and the methods employed are described in full elsewhere. ${ }^{10}$ A selection of 